\title{
Exp-Function Method for Generalized Travelling Solutions of Calogero-Degasperis-Fokas Equation
}

\author{
Syed Tauseef Mohyud-Din, Muhammad Aslam Noor, and Asif Waheed \\ Department of Mathematics, COMSATS Institute of Information Technology, Islamabad, Pakistan \\ Reprint requests to S. T. M.-D.; syedtauseefs@hotmail.com
}

Z. Naturforsch. 65a, 78 - 84 (2010); received June 19, 2008 / revised October 13, 2008

\begin{abstract}
In this paper, we apply a relatively new technique which is called the exp-function method to construct generalized solitary and periodic solutions of Calogero-Degasperis-Fokas (CDF) equation which plays a very important role in mathematical physics, applied and engineering sciences. The suggested algorithm is quite efficient and is practically well suited for use in these problems. Numerical results clearly indicate the reliability and efficiency of the proposed method.
\end{abstract}

Key words: Exp-Function Method; Calogero-Degasperis-Fokas (CDF) Equation; Nonlinear Partial Differential Equations.

\section{Introduction}

Many problems in natural and engineering sciences are modeled by nonlinear partial differential equations. These equations arise in a number of scientific models such as the propagation of shallow water waves, long wave and chemical reaction-diffusion models, astronomy, fluid mechanics, nonlinear optics, plasma physics and beam theory, see [1-13] and the references therein. A substantial amount of work has been invested for solving the governing equations of these physical models. Several techniques including method of characteristic, Riemann invariants, combination of waveform relaxation and multi grid, periodic multi grid wave form, hyperbolic function, double parameter hypothesis, inverse scattering transform, homogeneous balance and Jacobi elliptic have been used for the solutions of such problems, see $[5,8-11]$ and the references therein. Most of these techniques encounter the inbuilt deficiencies and involve huge computational work. He and $\mathrm{Wu}[2,12,13]$ developed the expfunction method to seek the solitary, periodic and compacton like solutions of nonlinear differential equations. The method has been implemented on Kortewegde Vries (KdV), high-dimensional nonlinear evolution equation, Burgers equations, combined $\mathrm{KdV}$ and modified (m)KdV, Hybrid-Lattice system and discrete $\mathrm{mKdV}$ lattice, Kuramoto-Sivashinsky and Boussinesq equations, and master partial differential equations, see $[1-4,6,7,12,14-18]$ and the references therein. Bekir and Boz [19] gave a historical remark on the exp-function method and the last development of the proposed technique was given by the originators of this method [4, 13,20]. Xu [21] and Zhang [22] explicitly showed the effectiveness of the exp-function method. This clearly indicates that the exp-function method is very effective and reliable. The basic motivation of this paper is to apply this reliable technique on a special nonlinear partial differential equation which is called the Calogero-Degasperis-Fokas (CDF) equation and was first introduced by Özer [10]. The CDF equation is of the form:

$$
\begin{aligned}
u_{t} & +\frac{1}{4} u_{x x x}-\frac{3}{4} \frac{u_{x} u_{x x}}{u}+\frac{3}{8} \frac{u_{x}^{3}}{u^{2}} \\
& +\frac{3}{8} u_{x}\left(\alpha u^{2}+\frac{\beta}{u^{2}}+\gamma\right)=0 .
\end{aligned}
$$

It has been investigated by different researchers due to its mathematical importance and its applications in applied sciences; see [10] and the references therein. It is observed that the exp-function method is very convenient and effective for constructing generalized solitary and periodic solutions of Calogero-DegasperisFokas (CDF) equation. Numerical results reveal the efficiency and accuracy of the proposed algorithm. The exp-function method can be viewed as an alternative to the existing mathematical techniques.

\section{Exp-Function Method}

We consider the general nonlinear PDE of the type

$$
P\left(u, u_{t}, u_{x}, u_{t t}, u_{x x}, u_{x x x x}, \ldots\right)=0 .
$$


Using a transformation

$$
\eta=k x+\omega t
$$

where $k$ and $\omega$ are constants, we can rewrite (2) in the following nonlinear ODE:

$$
Q\left(u, u^{\prime}, u^{\prime \prime}, u^{\prime \prime \prime}, u^{(i v)}, \ldots\right)=0,
$$

where the prime denotes derivation with respect to $\eta$. According to exp-function method, which was developed by $\mathrm{He}$ and $\mathrm{Wu}[2]$, we assume that the wave solution can be expressed in the following form:

$$
u(\eta)=\frac{\sum_{n=-c}^{d} a_{n} \exp [n \eta]}{\sum_{m=-p}^{q} b_{m} \exp [m \eta]}
$$

where $p, q, c$, and $d$ are positive integers which are known to be further determined, $a_{n}$ and $b_{m}$ are unknown constants. We can rewrite (5) in the following equivalent form:

$$
u(\eta)=\frac{a_{c} \exp [c \eta]+\ldots+a_{-d} \exp [-d \eta]}{b_{p} \exp [p \eta]+\ldots+b_{-q} \exp [-q \eta]} .
$$

This equivalent formulation plays an important and fundamental part for finding the analytic solution of problems. To determine the value of $c$ and $p$, we balance the linear term of highest order in (4) with the highest order nonlinear term. Similarly, to determine the value of $d$ and $q$, we balance the linear term of lowest order in (4) with lowest order nonlinear term.

\section{Numerical Applications}

In this section, we apply the exp-function method developed by $\mathrm{He}$ and $\mathrm{Wu}$ to construct generalized solitary and periodic solutions of the CalogeroDegasperis-Fokas (CDF) equation.

Example 1 [13]: Consider the general form of Calogero-Degasperis-Fokas (CDF) equation, as given in (1) as follows:

$$
\begin{aligned}
u_{t} & +\frac{1}{4} u_{x x x}-\frac{3}{4} \frac{u_{x} u_{x x}}{u}+\frac{3}{8} \frac{u_{x}^{3}}{u^{2}} \\
& +\frac{3}{8} u_{x}\left(\alpha u^{2}+\frac{\beta}{u^{2}}+\gamma\right)=0
\end{aligned}
$$

where $\alpha, \beta$, and $\gamma$ are arbitrary constants.
Introducing a transformation as $\eta=k x+\omega t$, we can covert (1) into an ordinary differential equation

$$
\begin{aligned}
8 \omega u^{\prime} & +2 k^{3} u^{\prime \prime \prime}-6 k^{3} \frac{u^{\prime} u^{\prime \prime}}{u}+3 k^{3} \frac{u^{\prime 3}}{u^{2}} \\
& +3 k u^{\prime}\left(\alpha u^{2}+\beta \frac{1}{u^{2}}+\gamma\right)=0
\end{aligned}
$$

where the prime denotes the derivative with respect to $\eta$. The solution of (7) can be expressed in the form (6)

$$
u(\eta)=\frac{a_{c} \exp [c \eta]+\ldots+a_{-d} \exp [-d \eta]}{b_{p} \exp [p \eta]+\ldots+b_{-q} \exp [-q \eta]}
$$

To determine the value of $c$ and $p$, we balance the linear term of highest order in (7) with the highest order nonlinear term

$$
u^{\prime \prime \prime}=\frac{c_{1} \exp [(7 p+c) \eta]+\cdots}{c_{2} \exp [8 p \eta]+\cdots}
$$

and

$$
\begin{aligned}
u^{2} u^{\prime} & =\frac{c_{3} \exp [(p+3 c) \eta]+\cdots}{c_{4} \exp [4 p \eta]+\cdots} \\
& =\frac{c_{3} \exp [(5 p+3 c) \eta]+\cdots}{c_{4} \exp [8 p \eta]+\cdots}
\end{aligned}
$$

where $c_{i}$ are determined coefficients only for simplicity; balancing the highest order of exp-function in (8) and (9), we have

$$
5 p+3 c=7 p+c,
$$

which in turn gives

$$
p=c \text {. }
$$

To determine the value of $d$ and $q$, we balance the linear term of lowest order in (6) with the lowest order nonlinear term

$$
u^{\prime \prime \prime}=\frac{\cdots+d_{1} \exp [(-d-7 q) \eta]}{\cdots+d_{2} \exp [-8 q \eta]}
$$

and

$$
\begin{aligned}
u^{\prime} u^{\prime \prime} & =\frac{\cdots+d_{3} \exp [(-q-3 d) \eta]}{\cdots+d_{4} \exp [-4 q \eta]} \\
& =\frac{\cdots+d_{3} \exp [(-3 d-5 q) \eta]}{\cdots+d_{4} \exp [-8 q \eta]}
\end{aligned}
$$


where $d_{i}$ are determined coefficients only for simplicity. Now, balancing the lowest order of exp-function in (12) and (13), we have

$$
-7 q-d=-5 q-3 d,
$$

which in turn gives

$$
q=d
$$

Case i. We can freely choose the values of $c$ and $d$, but we will illustrate that the final solution does not strongly depend upon the choice of values of $c$ and $d$. For simplicity, we set $p=c=1$ and $q=d=1$, then the trial solution (6) reduces to

$$
u(\eta)=\frac{a_{1} \exp [\eta]+a_{0}+a_{-1} \exp [-\eta]}{b_{1} \exp [\eta]+a_{0}+b_{-1} \exp [-\eta]} .
$$

Substituting (16) into (7), we have

$$
\begin{aligned}
& \frac{1}{A}\left(c_{5} \exp [5 \eta]+c_{4} \exp [4 \eta]+c_{3} \exp [3 \eta]\right. \\
& \quad+c_{2} \exp [2 \eta]+c_{1} \exp [\eta]+c_{0}+c_{-1} \exp [-\eta] \\
& \quad+c_{-2} \exp [-2 \eta]+c_{-3} \exp [-3 \eta] \\
& \left.\quad+c_{-4} \exp [-4 \eta]+c_{-5} \exp [-5 \eta]\right)=0,
\end{aligned}
$$

where $A=8\left(\exp [\eta]+b_{0}+b_{-1} \exp [-\eta]\right)^{4}\left(a_{1} \exp [\eta]+\right.$ $\left.a_{0}+a_{-1} \exp [\eta]\right)^{2}, c_{i}(u=-5,-4, \ldots, 4,5)$ are constants obtained by the simulation software Maple 7 . Equating the coefficients of $\exp [n \eta]$ to be zero, we obtain

$$
\begin{aligned}
& c_{-5}=0, c_{-4}=0, c_{-3}=0, c_{-2}=0, c_{-1}=0, \\
& c_{0}=0, c_{1}=0, c_{2}=0, c_{3}=0, c_{4}=0, c_{5}=0 .
\end{aligned}
$$

Solution of (18) will yield

$$
\begin{aligned}
a_{-1}= & a_{1} b_{0}{ }^{2}\left(a_{1} 8 \alpha^{2}+2 a_{1}{ }^{6} k^{2} \alpha-2 \beta \alpha a^{4}+a_{1}{ }^{4} k^{4}\right. \\
& \left.+2 a_{1}{ }^{2} \beta k^{2}+\beta^{2}\right)\left[4\left(-\alpha a_{1}{ }^{4}+\beta+k^{2} a_{1}{ }^{2}\right)^{2}\right]^{-1}, \\
b_{0}= & b_{0}, \\
b_{-1}= & b_{0}{ }^{2}\left(a_{1}{ }^{8} \alpha^{2}+2 a_{1}{ }^{6} k^{2} \alpha-2 \beta \alpha a_{1}{ }^{4}+a_{1}{ }^{4} k^{4}\right. \\
& \left.+2 a_{1}{ }^{2} \beta k^{2}+\beta^{2}\right)\left[4\left(-\alpha a_{1}{ }^{4}+\beta+k^{2} a_{1}{ }^{2}\right)^{2}\right]^{-1}, \\
a_{1}= & a_{1}, \\
\omega= & -\frac{1}{8} \frac{k\left(2 k^{2} a_{1}{ }^{2}+3 \gamma a_{1}{ }^{2}+3 \alpha a_{1}{ }^{4}+3 \beta\right)}{a_{1}{ }^{2}}, \\
a_{0}= & \frac{a_{1} b_{0}\left(-\alpha a_{1}{ }^{4}+\beta+k^{2} a_{1}{ }^{2}\right)}{-\alpha a_{1}{ }^{4}+\beta+k^{2} a_{1}^{2}} .
\end{aligned}
$$

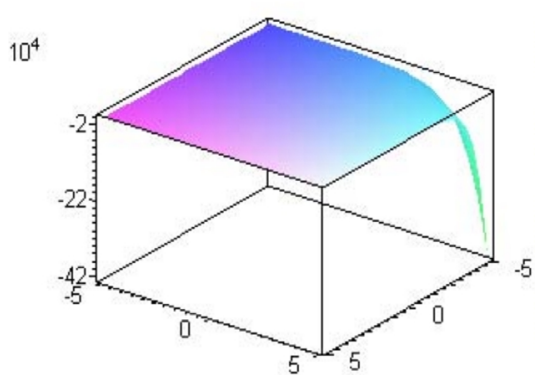

Fig. 1. Soliton solutions of (1), when $a_{1}=b_{0}=k=\beta=1$ and $\alpha=\gamma=2$.

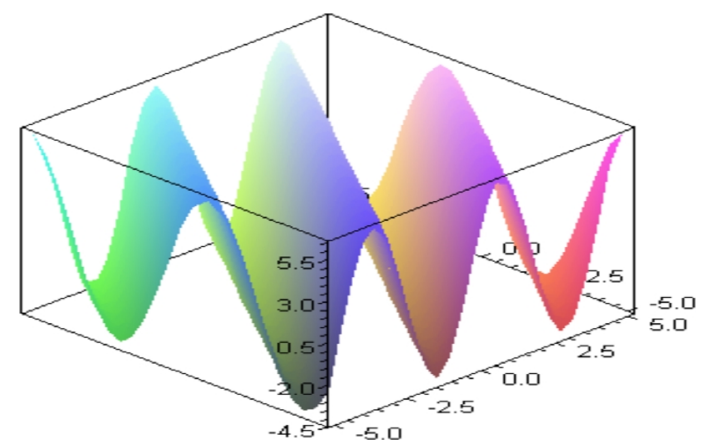

Fig. 2. Periodic solutions of (1), when $a_{1}=b_{0}=K=\alpha=1$ and $\beta=2, \gamma=3$.

We, therefore, obtained the following generalized solitary solution $u(x, t)$ of (1):

$u(x, t)=a_{1}+2 k^{2} a_{1}{ }^{3} b_{0}(\beta-\alpha)\left\{\left(-\alpha a_{1}{ }^{4}+\beta+k^{2} a_{1}{ }^{2}\right)^{2}\right.$
$\cdot\left(\exp [k x+\omega t]+b_{0}\right)+\frac{1}{4} b_{0}{ }^{2}\left(a_{1}{ }^{8} \alpha^{2}+2 a_{1}{ }^{6} k^{2} \alpha\right.$
$\left.\left.-2 \beta \alpha a_{1}{ }^{4}+a_{1}{ }^{4} k^{4}+2 a_{1}{ }^{2} \beta k^{2}+\beta^{2}\right)(\exp [-k x-\omega t])\right\}^{-1}$,

where $\omega=-\frac{1}{8} k\left(2 k^{2} a_{1}{ }^{2}+3 \gamma a_{1}{ }^{2}+3 \alpha a_{1}{ }^{4}+3 \beta\right) / a_{1}{ }^{2}$ and $a_{1}, b_{0}, \alpha, \beta, \gamma$, and $k$ are real numbers.

Figure 1 shows the soliton solutions of (1), when $a_{1}=b_{0}=k=\beta=1$ and $\alpha=\gamma=2$. In case $k$ is an imaginary number, the obtained soliton solution can be converted into periodic solution or compact-like solution. Therefore, we write $k=\mathrm{i} K$, consequently, (20) becomes

$$
\begin{aligned}
& u(x, t)=a_{1}-2 K^{2} a_{1}{ }^{3} b_{0}(\beta-\alpha)\left\{\left(-\alpha a_{1}{ }^{4}+\beta\right.\right. \\
& \left.+K^{2} a_{1}{ }^{2}\right)^{2}\left(\exp [-\mathrm{i} k x+\omega t]+b_{0}\right)+\frac{1}{4} b_{0}{ }^{2}\left(a_{1}{ }^{8} \alpha^{2}\right. \\
& \left.-2 a_{1}{ }^{6} K^{2} \alpha-2 \beta \alpha a_{1}{ }^{4}+a_{1}{ }^{4} K^{4}-2 a_{1}{ }^{2} \beta K^{2}+\beta^{2}\right) \\
& \cdot(\exp [-\mathrm{i} k x-\omega t])\}^{-1},
\end{aligned}
$$


where $\omega=-\frac{1}{8} \mathrm{i} K\left(-2 K^{2} a_{1}^{2}+3 \gamma a_{1}^{2}+3 \alpha a_{1}^{4}+3 \beta\right) / a_{1}^{2}$ and $a_{1}, b_{0}, \alpha, \beta, \gamma$, and $K$ are real numbers. If we search for periodic solution or compact-like solution, the imaginary part in (21) must be zero that requires, therefore (21) becomes

$$
u(x, t)=a_{1} \frac{\left[\begin{array}{l}
\cos (K x+\omega t)\left(\begin{array}{l}
32 m^{4} b_{0}+16 m^{2}(K x+\omega t) b_{0}{ }^{2} p+8 m^{2} b_{0}{ }^{3} p-32 K^{2} \alpha_{1}{ }^{2} b_{0} \beta m^{2} \\
-8 K^{2} a_{1}{ }^{2} b_{0}{ }^{3} \beta p+32 K^{2} a_{1}{ }^{2} b_{0} \alpha m^{2}+8 K^{2} a_{1}{ }^{2} b_{0}{ }^{3} \alpha p
\end{array}\right)+16 m^{4} b_{0}{ }^{2} \\
16 m^{4}-8 m^{2} b_{0}{ }^{2} p+b_{0}{ }^{4} p^{2}-32 K^{2} a_{1}{ }^{2} b_{0}{ }^{2} \beta m^{2}+32 K^{2} a_{1}{ }^{2} b_{0}{ }^{2} \alpha m^{2}
\end{array}\right]}{(K x+\omega t)\left(32 m^{4} b_{0}+16 m^{2} b_{0}{ }^{2} p+8 m^{2} b_{0} 3 p\right)+16 m^{4} b_{0}{ }^{2}+16 m^{4}-8 m^{2} b_{0}{ }^{2} p+b_{0}{ }^{4} p^{2}},
$$

where

$$
\begin{aligned}
m= & -\alpha a_{1}{ }^{4}+\beta+k^{2} a_{1}{ }^{2}, \\
p= & a_{1}{ }^{8} \alpha^{2}+2 a_{1}{ }^{6} k^{2} \alpha-2 \beta \alpha a_{1}{ }^{4}+a_{1}{ }^{4} k^{4} \\
& +2 a_{1}{ }^{2} \beta k^{2}+\beta^{2}, \\
\omega= & \frac{1}{8} \frac{k\left(2 k^{2} a_{1}{ }^{2}+3 \gamma a_{1}{ }^{2}+3 \alpha a_{1}{ }^{4}+3 \beta\right)}{a_{1}{ }^{2}},
\end{aligned}
$$

which is a periodic solution of (1). See Figure 2. to

Case ii. If $p=c=2$, and $q=d=1$, than (6) reduces

$$
u(\eta)=\frac{a_{2} \exp [2 \eta]+a_{1} \exp [\eta]+a_{0}+a_{-1} \exp [-\eta]}{b_{2} \exp [2 \eta]+b_{1} \exp [\eta]+b_{0}+b_{-1} \exp [-\eta]}
$$

Proceeding as before, we obtain

$$
\begin{aligned}
& a_{-1}=a_{-1}, \quad a_{0}=a_{-1}, \quad a_{1}=a_{2}, \quad a_{2}=a_{2}, \\
& \alpha=-4 k^{2} /\left(-a_{2}+a_{-1}\right)^{2}, \quad b_{2}=b_{0}=1, \quad a_{2} \neq a_{-1}, \\
& \beta=4 a_{2}^{2} k^{2} a_{-1}{ }^{2} /\left(-a_{2}+a_{-1}\right)^{2} \text {, } \\
& \omega=\frac{1}{8} k\left(4 k^{2} a_{-1}^{2}-3 \gamma^{2} a_{2}^{2}+6 \gamma a_{-1} a_{2}-3 a_{-1}^{2} \gamma\right. \\
& \left.+4 k^{2} a_{2}^{2}+16 k^{2} a_{-1} a_{2}\right) /\left(-a_{2}+a_{-1}\right)^{2} \text {. }
\end{aligned}
$$

Hence we get the generalized solitary wave solutions of (1) as follows

$$
\begin{aligned}
& u(x, t)=\left\{a_{2} \exp [2 k x+2 \omega t]+a_{2} \exp [k x+\omega t]\right. \\
& \left.+a_{-1}+a_{-1} \exp [-k x-\omega t]\right\} /\{\exp [2 k x+2 \omega t] \\
& \left.+b_{1} \exp [k x+\omega t]+1+b_{-1} \exp [-k x-\omega t]\right\}
\end{aligned}
$$

where

$$
\begin{array}{r}
\omega=\frac{1}{8}\left\{k \left(4 k^{2} a_{-1}{ }^{2}-3 \gamma^{2} a_{2}{ }^{2}+6 \gamma a_{-1} a_{2}-3 a_{-1}{ }^{2} \gamma\right.\right. \\
\left.\left.+4 k^{2} a_{2}{ }^{2}+16 k^{2} a_{-1} a_{2}\right)\right\} /\left(-a_{2}+a_{-1}\right)^{2} .
\end{array}
$$

\section{Special cases}

For $\alpha=0,(1)$ can be written as

$u_{t}+\frac{1}{4} u_{x x x}-\frac{3}{4} \frac{u_{x} u_{x x}}{u}+\frac{3}{8} \frac{u_{x}^{3}}{u^{2}}+\frac{3}{8} u_{x}\left(\frac{\beta}{u^{2}}+\gamma\right)=0$.

Introducing a transformation as $\eta=k x+\omega t$, we can covert (26) into the ordinary differential equation

$$
\begin{aligned}
8 \omega u^{\prime} & +2 k^{3} u^{\prime \prime \prime}-6 k^{3} \frac{u^{\prime} u^{\prime \prime}}{u}+3 k^{3} \frac{u^{\prime 3}}{u^{2}} \\
& +3 k u^{\prime}\left(\beta \frac{1}{u^{2}}+\gamma\right)=0
\end{aligned}
$$

where the prime denotes the derivative with respect to $\eta$. The solution of (27) can be expressed in the form of (6)

$$
u(\eta)=\frac{a_{c} \exp [c \eta]+\ldots+a_{-d} \exp [-d \eta]}{b_{p} \exp [p \eta]+\ldots+b_{-q} \exp [-q \eta]} .
$$

Proceeding as before, from (9) to (10) we have

$$
p=c \text {. }
$$

Similarly, from (12) to (14) we have

$$
q=d
$$

Case iii. We can freely choose the values of $c$ and $d$, but we will illustrate that the final solution does not strongly depend upon the choice of values of $c$ and $d$. For simplicity, we set $p=c=1$ and $q=d=1$, then the trial solution, (6) reduces to (17)

$$
u(\eta)=\frac{a_{1} \exp [\eta]+a_{0}+a_{-1} \exp [-\eta]}{b_{1} \exp [\eta]+a_{0}+b_{-1} \exp [-\eta]} .
$$




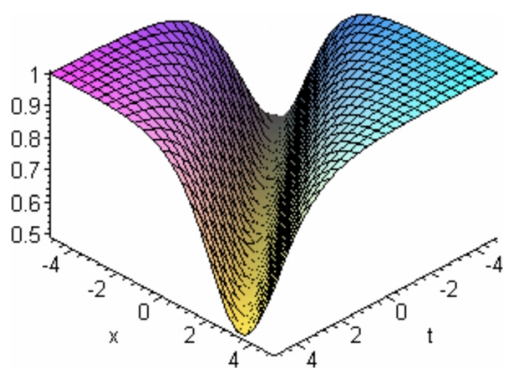

Fig. 3. Soliton solutions of (26), when $a_{1}=b_{0}=k=\gamma=$ $\beta=1$.

Substituting (17) into (27), we have

$$
\begin{aligned}
& \frac{1}{A}\left[c_{5} \exp [5 \eta]+c_{4} \exp [4 \eta]+c_{3} \exp [3 \eta]\right. \\
& \quad+c_{2} \exp [2 \eta]+c_{1} \exp [\eta]+c_{0} \\
& \quad+c_{-1} \exp [-\eta]+c_{-2} \exp [-2 \eta] \\
& \quad+c_{-3} \exp [-3 \eta]+c_{-4} \exp [-4 \eta] \\
& \left.\quad+c_{-5} \exp [-5 \eta]\right]=0
\end{aligned}
$$

where $A=8\left(\exp [\eta]+b_{0}+b_{-1} \exp [-\eta]\right)^{4}\left(a_{1} \exp [\eta]+\right.$ $\left.a_{0}+a_{-1} \exp [\eta]\right)^{2}, c_{i}(i=-5,-4, \ldots, 4,5)$ are constants obtained by Maple 7 . Equating the coefficients of $\exp [n \eta]$ to be zero, we obtain

$$
\begin{aligned}
& c_{-5}=0, \quad c_{-4}=0, \quad c_{-3}=0, \quad c_{-2}=0, \\
& c_{-1}=0, \quad c_{0}=0, \quad c_{1}=0, \quad c_{2}=0, \\
& c_{3}=0, \quad c_{4}=0, \quad c_{5}=0 .
\end{aligned}
$$

Solution of (31) will yield

$$
\begin{aligned}
& a_{-1}=\frac{1}{4} a_{1} b_{0}^{2}, \quad b_{0}=b_{0}, \quad a_{1}=a_{1}, \\
& \omega=-\frac{1}{8} \frac{k\left(2 k^{2} a_{1}^{2}+3 \gamma a_{1}^{2}+3 \beta\right)}{a_{1}^{2}}, \\
& a_{0}=\frac{a_{1} b_{0}\left(\beta-k^{2} a_{1}^{2}\right)}{\beta+k^{2} a_{1}^{2}}, \quad b_{-1}=\frac{1}{4} b_{0}^{2} .
\end{aligned}
$$

We, therefore, obtained the following generalized soliton solution $u(x, t)$ of (26):

$$
\begin{aligned}
& u(x, t)=a_{1}\left(1-2 k^{2} a_{1}^{2} b_{0}\right) /\left[\left(k^{2} a_{1}^{2}+\beta\right)\right. \\
& \left.\cdot\left(\exp [k x+\omega t]+b_{0}+\frac{1}{4} b_{0}^{2} \exp [-k x-\omega t]\right)\right],
\end{aligned}
$$

where $\omega=-\frac{1}{8} k\left(2 k^{2} a_{1}^{2}+3 \gamma a_{1}^{2}+3 \beta\right) / a_{1}^{2}$ and $a_{1}, b_{0}$, $\beta, \gamma$, and $k$ are real numbers.

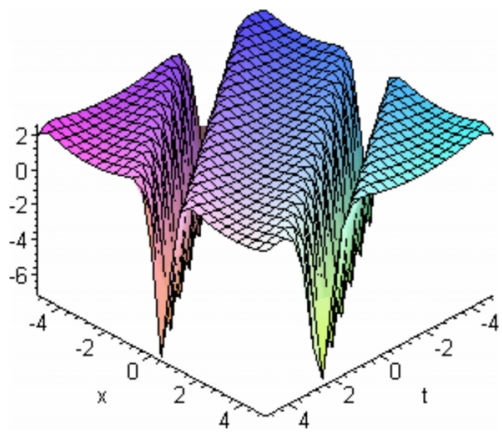

Fig. 4. Periodic solutions of (26), when $a_{1}=b_{0}=K=1$ and $\beta=2, \gamma=3(\beta \neq 1)$.

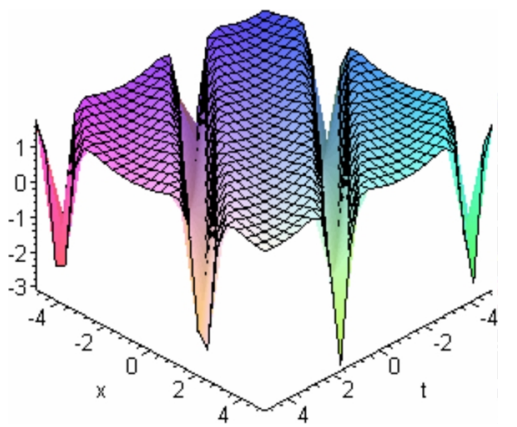

Fig. 5. Periodic solutions of (26), when $a_{1}=b_{0}=K=1$ and $\beta=3, \gamma=1(\beta \neq 1)$.

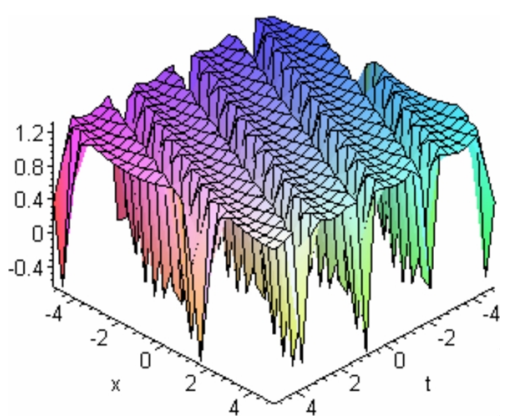

Fig. 6. Periodic solutions of (26), when $a_{1}=b_{0}=K=1$ and $\beta=6, \gamma=1(\beta \neq 1)$.

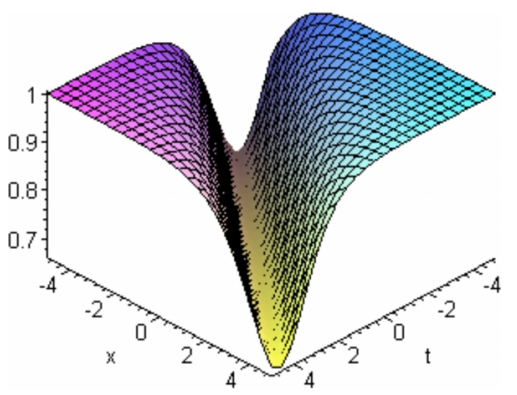

Fig. 7. Soliton solutions of (26) for case ii, when $a_{2}=b_{1}=$ $k=1$, and $\beta=\gamma=2$. 
Figure 3 shows the soliton solutions of (26), when $a_{1}=b_{0}=k=\gamma=\beta=1$. In case $k$ is an imaginary number, the obtained soliton solution can be converted into a periodic solution or compact-like solution. Therefore, we write $k=\mathrm{i} K$, consequently, (33) becomes

$$
u(x, t)=a_{1}\left[1+2 K^{2} a_{1}^{2} b_{0} /\left\{\left(-K^{2} a_{1}^{2}+\beta\right)\left(\exp [\mathrm{i} K x+\omega t]+b_{0}+\frac{1}{4} b_{0}^{2} \exp [-\mathrm{i} K x-\omega t]\right)\right\}\right],
$$

where $\left.\omega=-\frac{1}{8} \mathrm{i} K^{2} a_{1}{ }^{2}+3 \gamma a_{1}{ }^{2}+3 \beta\right) / a_{1}{ }^{2}$. If we search for a periodic solution or compact-like solution, the imaginary part in (34) must be zero, therefore (34) becomes

$$
u(x, t)=a_{1} \frac{\left(\begin{array}{l}
\cos (K x+\theta t)\left(-16 K^{2} a_{1}{ }^{2} b_{0}{ }^{2} \cos (K x+\theta t)+32 \beta b_{0}+16 \beta b_{0}{ }^{2} \cos (K x+\theta t)+8 \beta b_{0}{ }^{3}\right) \\
+24 K^{2} a_{1}{ }^{2} b_{0}{ }^{2}-16 K^{2} a_{1}-K^{2} a_{1}{ }^{2} b_{0}{ }^{4}+8 \beta b_{0}{ }^{3}+16 \beta+\beta b_{0}{ }^{4}
\end{array}\right)}{\left(-K^{2} a_{1}{ }^{2}+\beta\right)\left(\cos (K x+\theta t)\left(32 b_{0}+16 b_{0}{ }^{2} \cos (K x+\theta t)+8 b_{0}^{3}\right)+16+b_{0}{ }^{4}+8 b_{0}{ }^{2}\right)}
$$

where $\theta=K\left(2 K^{2} a_{1}^{2}-3 \gamma a_{1}^{2}-3 \beta\right) / 8 a_{1}^{2}$.

It is noticed that the periodic solution is stable for a long range of values of $\gamma$ while wave form of the periodic solution changes with the values of $\beta$ as given in Figures 4-6.

Case iv. If $p=c=2$, and $q=d=1$, then (6) reduces to $(23)$

$$
u(\eta)=\frac{a_{2} \exp [2 \eta]+a_{1} \exp [\eta]+a_{0}+a_{-1} \exp [-\eta]}{b_{2} \exp [2 \eta]+b_{1} \exp [\eta]+b_{0}+b_{-1} \exp [-\eta]}
$$

There are some free parameters in (24). If we set $b_{2}=1$, for simplicity, the trial-function (24) is simplified as follows:

$$
u(\eta)=\frac{a_{2} \exp [2 \eta]+a_{1} \exp [\eta]+a_{0}+a_{-1} \exp [-\eta]}{\exp [2 \eta]+b_{1} \exp [\eta]+b_{0}+b_{-1} \exp [-\eta]}
$$

Proceeding as before, we obtain

$$
\begin{aligned}
& a_{-1}=0, \quad a_{0}=\frac{1}{4} a_{2} b_{1}^{2}, \quad a_{1}=\frac{b_{1} a_{2}\left(\beta-k^{2} a_{2}^{2}\right)}{\beta+k^{2} a_{2}^{2}}, \\
& a_{2}=a_{2}, \quad b_{1}=0, \quad b_{-1}=0, \quad b_{0}=\frac{1}{4} b_{1}^{2}, \\
& \omega=-\frac{1}{8} \frac{k\left(3 \beta+2 k^{2} a_{2}^{2}+3 \gamma a_{2}^{2}\right)}{a_{2}^{2}} .
\end{aligned}
$$

Hence we get the generalized solitary wave solution of (26) as follows:

$$
\begin{aligned}
& u(x, t)=a_{2}\left[1-2 k^{2} a_{2}{ }^{2} b_{1} /\left\{\left(-k^{2} a_{2}^{2}+\beta\right)\right.\right. \\
& \left.\left.\cdot\left(\exp [2 k x+2 \omega t]+b_{1} \exp [k x+\omega t]+\frac{1}{4} b_{1}^{2}\right)\right\}\right],
\end{aligned}
$$

where $\omega=-\frac{1}{8} k\left(3 \beta+2 k^{2} a_{2}^{2}+3 \gamma_{2}^{2}\right) / a_{2}^{2}$, and $a_{2}, b_{1}$, $\beta, \gamma$, and $k$ are real numbers hence we get similar result as in case iii (33).

Figure 7 shows soliton solutions of (26) for case ii, when $a_{2}=b_{1}=k=1$ and $\beta=\gamma=2$. In both cases (iii and iv), for different choices of $c, p, d$, and $q$ we get the same soliton solution which clearly illustrate that the final solution does not strongly depends upon these parameters.

Remark: It is worth mentioning that the transformation $k=\mathrm{i} k$ which is used to transform the solitary solution to a periodic or compacton-like solution was first proposed by $\mathrm{He}$ and $\mathrm{Wu}$ [2] and is called the $\mathrm{He}$ Wu transformation. Moreover, the interpretation of this transformation is given by $\mathrm{He}[1]$.

\section{Conclusion}

In this paper, we applied the exp-function method with computerized symbolic computation, developed by $\mathrm{He}$ and $\mathrm{Wu}$ [2], to obtain the generalized solitary and periodic solution of the Calogero-DegasperisFokas (CDF) equation. The results are very promising and encouraging. Hence, we conclude that the expfunction method is a very effective and powerful mathematical tool for finding solitary and periodic solutions of Calogero-Degasperis-Fokas (CDF) equations.

\section{Acknowledgement}

The authors are highly grateful to the referee for his/her very constructive comments. We would like to thank Prof. Dr. Ji-Huan He for the guidance and Dr. S. M. Junaid Zaidi, Rector CIIT, for providing excellent research facilities and environment. 
[1] J. H. He, Int. J. Mod. Phys. B 22, 3487 (2008).

[2] J.H. He and X.H. Wu, Chaos, Solitons, and Fractals 30, 700 (2006)

[3] J.H. He and M. A. Abdou, Chaos, Solitons, and Fractals 34, 1421 (2007).

[4] J.H. He and L.N. Zhang, Phys. Lett. A 372, 1044 (2008).

[5] C. Lubich and A. Ostermann, BIT 27, 216 (1987).

[6] M. A. Noor, S.T. Mohyud-Din, and A. Waheed, J. Appl. Math. Computg. 29, 81 (2008).

[7] M. A. Noor, S. T. Mohyud-Din, and A. Waheed, Acta Applnda. Mathmtce. 104, 131 (2008).

[8] M. A. Noor and S. T. Mohyud-Din, Math. Prob. Eng. 2008, 11 (2008).

[9] M. A. Noor and S.T. Mohyud-Din, Acta Applnda. Mathmtce. (in press).

[10] T. Özer, Chaos, Solitons, and Fractals 39, 1371 (2009).

[11] S. Vandewalle and R. Piessens, Appl. Numer. Math. 8, 149 (1991).
[12] X. H. Wu and J.H. He, Chaos, Solitons, and Fractals 38, 903 (2008).

[13] X. H. Wu and J.H. He, Comput. Math. Appl. 54, 966 (2007).

[14] M. A. Abdou, A. A. Soliman, and S. T. Basyony, Phys. Lett. A 369, 469 (2007).

[15] S. A. El-Wakil, M. A. Madkour, and M. A. Abdou, Phys. Lett. A 369, 62 (2007).

[16] E. Yusufoglu, Phys. Lett. A. 372, 442 (2008).

[17] S. D. Zhu, Inter. J. Nonlin. Sci. Numer. Simul. 8, 461 (2007).

[18] S. D. Zhu, Inter. J. Nonlin. Sci. Numer. Simul. 8, 465 (2007).

[19] A. Bekir and A. Boz, Int. J. Nonlin. Sci. Numer. Simul. 8, 505 (2007).

[20] X. W. Zhou, Y. X. Wen, and J. H. He, Int. J. Nonlin. Sci. Numer. Simul. 9, 301 (2008).

[21] F. Xu, Z. Naturforsch. 62a, 685 (2007).

[22] S. Zhang, Z. Naturforsch. 62a, 689 (2007). 